\title{
ADDITIVE AND MULTIPLICATIVE NOISE REMOVAL BASED ON ADAPTIVE WAVELET TRANSFORMATION USING CYCLE SPINNING
}

\author{
Asem Khmag, Abd Rahman Ramli, \\ Syed Abdul Rahman Al-Haddad and Shaiful Jahari Bin Hashim \\ Department of Computer and Communication Systems, \\ Faculty of Engineering, Universiti Putra Malaysia (43400), Serdang, Malaysia
}

Received 2013-12-01; Revised 2013-12-27; Accepted 2013-12-27

\begin{abstract}
The need for image restoration is encountered in many practical applications. For instance, distortion due to Additive White Gaussian Noise (AWGN) or in some cases the multiplicative (speckle) one can be caused by poor quality image acquisition. Wavelet denoising attempts to remove these types of noise present in the signal while preserving the signal characteristics, regardless of its frequency content. A newly developed method based on the wavelet transform (semi-soft thresholding) appears promising, though there is little practical guidance on its use. The results that are obtained by the experiments are compared with traditional additive noise methods such as Sureshrink, Block Method 3 Dimensions (BM3D) and Speckle noise reduction methods as Lee filter, linear scaling filter (Lsmv). Furthermore, Cycle Spinning technique is implemented in order to enhance the quality of the denoised estimates. It has been found that the proposed method achieves better enhancement and restoration of the image while preserving high frequency features of the noiseless image. Moreover, the proposed algorithm matches the quality of the best redundant approaches, while maintaining a high computational efficiency and a low CPU/memory consumption.
\end{abstract}

Keywords: Additive Noise, Speckle Noise, Wavelet, BM3D, Cycle Spinning

\section{INTRODUCTION}

An image can be defined as a two-dimensional function $\mathrm{f}(\mathrm{x}, \mathrm{y})$, where $\mathrm{x}$ and $\mathrm{y}$ are spatial coordinates and the amplitude of $\mathrm{f}$ function at any pair of coordinates $(\mathrm{x}, \mathrm{y})$ is called the gray level or intensity of the image at that point. This image is often corrupted by noise since its acquisition or transmission (Narbada and Bhagwan, 2013).

In recent years, the image denoising problem has been widely studied and two main approaches have been developed: The variational methods (Aujol and Chambolle, 2005; Rudin et al., 1992; Chambolle, 2004; Osher et al., 2003; Gilboa et al., 2006) and wavelet techniques (Meyer, 2002; Donoho and Johnstone, 1994; 1995a; Chang et al., 2000; Chambolle et al., 1998; Daubechies, 1990).
Sankar et al. (2012) proposed a new method to extract respiratory signal from the recorded abdomen movement using hybrid of neural and fuzzy inference system.

Also, there is a rapidly increasing interest in designing hybrid methods using both wavelet shrinkage and the Total Variation (TV) denoising methods (Banazier and Yasser, 2011), but this method has shortcoming in the regions near to the edges in the image and its valid for speckle noise only. Khmag et al. (2013), studied and summarized some standard methods that used the adaptive filtering technique to reduce the additive white Gaussian noise impacts.

Durand and Froment (2003) proposed a hybrid method to remove the pseudo-Gibbs phenomenon by replacing the thresholded coefficients by values that minimized TV; this method presents some drawbacks

Corresponding Author: Asem Khmag, Department of Computer and Communication Systems, Faculty of Engineering, Universiti Putra Malaysia (43400), Serdang, Malaysia 
where it gave extra sharpness especially in high level scale of noises. In the 1D signal, the various artifacts mixed with cannot be filtered directly because they pass through the body and turn into an interference component. Balaiah and Ilavennila (2012) found that EEG is subjected to noise signal and it is contaminated. Then the noise is removed by means of Adaptive filter and Neuro-fuzzy filter. A new approach for Digital Imaging and Communications in Medicine (DICOM) image is done by applying contrast stretching and anisotropic diffusion where denoising of multiplicative noise is carried out and the level of contrast is improved (Yasmin et al., 2012).

The classical wavelet-based denoising method was proposed by Donoho and Johnstone (1995a), both types of threshding that were presented in this study have a limitations such as blurring and over smoothing in the reconstructed image. In addition, it is a typical nonlinear wavelet threshold shrinkage method, but the deficiency of such methods is that some details of the image are also removed while the image noise is reduced. Therefore, a lot of improved algorithms are proposed to solve this issue (Hsung et al., 2005; Qing-Wu et al., 2007), although they solve the problem of killing too many coefficients, but they still suffer from the time complexity where the use of Biorthogonal wavelet transformation leads to extra calculation and make it more complicated. In order to improve the efficiency of the image denoising techniques, Principal Component Analysis (PCA) is used, in which the removal of redundant and unwanted data is done. Applications such as Median Filtering and Adaptive thresholding are used for handling the variations in lighting and noise (Suganthy and Ramamoorthy, 2012). An image denoising algorithm based on Gaussian scale mixture model and Bayes Least Squares estimator (BLS-GSM) was also developed (Portilla et al., 2003), those methods and studies came up with a good results in the qualitative and quantitative points of view, but when the image is a symmetric or with periodic texture, the efficiency decreases.

Luisier et al. (2007) introduced a new interscale SUREbased approach to Orthonormal Wavelet image denoising (OWT-SURELET), the drawback of this method is that for Orthogonal wavelets, the same scaling filter is used for decomposition (analysis) and reconstruction (synthesis) the signal, it causes of delay analysis of the image. A recent study by Ladan et al. (2013) found that the best wavelet parameters were identified as those that provide less RMSE and did not change the details of the original image; this study is valid especially for data related to space and satellite images where it used for GIS applications in forest and urban areas.

In this study, we are presenting a new image denoising scheme that performs better than the classical algorithms in both types of noise whether it is additive or multiplicative noise. This method is based on semisoft thresholding in order to remove the noise and artifact from contaminated images. The criteria of the noise removal problem depend on the noise type by which the image is corrupted, where Gaussian and Spackle noises are the main types of noise that the proposed algorithm deals with.

The paper is organized as follows. The image denoising problem is discussed in section 2. Section 3 discusses 2D discrete wavelet transformation and section 4 discusses the proposed method. Experimental results and discussion is presented in section 5. Finally, this study is concluded in section 6 .

\subsection{The Image Denoising Problem}

In this section, the image denoising and some of the preliminary issues such as the assumptions about the noise in images, noise models and types of noises will be discussed.

\subsection{Image Degradation and Restoration}

A noisy image as additive type in spatial domain is modeled by Equation (1):

$\hat{\mathrm{u}}=\mathrm{u}+\mathrm{w}$

where, $\hat{u}$ is the observed image, $\mathrm{u}$ is the unknown original image and $\mathrm{w}$ is assumed to be an independent and identically distributed random white Gaussian noise with zero mean and finite variance $\sigma_{n}^{2}$. Where the noise $\mathrm{w}_{\mathrm{ij}} \sim \mathrm{W}\left(0, \sigma^{2}\right)$. Figure 1 show the degradation process of the image that contaminated with additive noise $\mathrm{w}$, the denoised image $\hat{u}$ is desired to be as close as possible to the original image $u$.

Multiplicative noise model is another most frequently investigated noise model. Different from the Additive white Gaussian Noise (AGWN), the primary characteristic of the multiplicative noise is that a portion of the pixels within an image are corrupted by random-value, while the remaining pixels are unaffected. The mathematical expression of the multiplicative noise model (x1) is given by Shamik et al. (2011) as follow Equation (2):

$$
x_{1}[i, j]=\left\{\begin{array}{l}
\eta_{1}[i, j] \quad \text { with probability } p 1 \\
x_{0}[i, j] \quad \text { with probability } 1-p 1
\end{array}\right.
$$




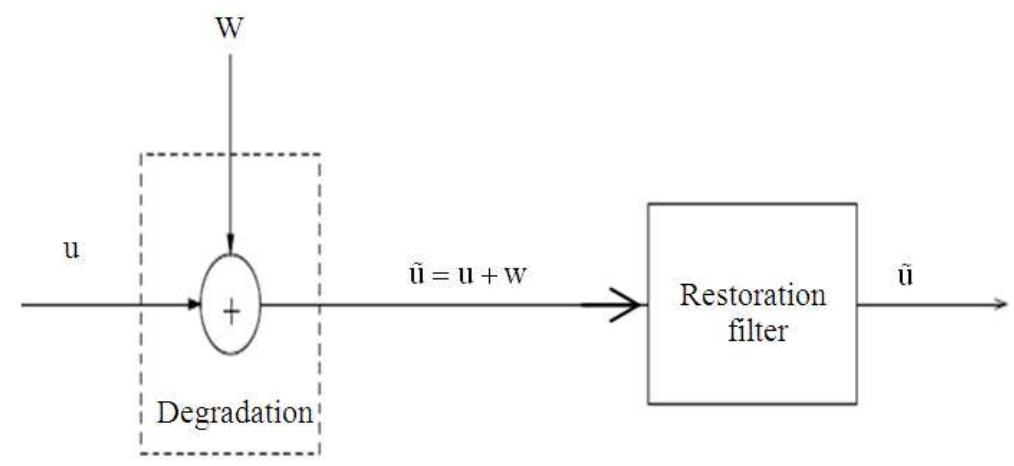

Fig. 1. The degradation process of the image

where, $\eta_{1}[i, j]$ denotes the corrupted pixel in the noisy image $\eta_{1}$, whose pixel values are uniformly distributed within [Lmin; Lmax]. Usually Lmin $=0$; Lmax $=255$ for 8-bit gray-scale images. $\mathrm{p} 1$ is the probability of a pixel being corrupted by the multiplicative noise.

\subsection{Noise Model}

Noise can be additive or multiplicative. Gaussian noise can be classified as additive noise. The noisy image looks soft and slightly blurry, where each pixel in the image will be changed from its original values by a small amount. The model of additive noise can be written as Equation (3):

$$
\mathrm{F}(\mathrm{x}, \mathrm{y})=\mathrm{O}(\mathrm{x}, \mathrm{y})+\beta(\mathrm{x}, \mathrm{y})
$$

where, $F(x, y)$ is the observed image function, $O(x, y)$ is the original image and $\beta(x, y)$ represent the signal independent additive random noise.

The Gaussian noise has a normal (Gaussian) probability function as Equation (4):

$$
\mathrm{PDF}_{\text {Gaussian }}=\frac{1}{\sqrt{2 \pi \sigma^{2}}} \mathrm{e}^{\frac{-(\mathrm{g}-\mu)}{2 \sigma^{2}}}
$$

where, $g$ is gray level, $\mu$ is mean, $\sigma$ is standard deviation.

In some cases, voltage spikes in the equipment can be the cause of the noise in the circuit, where the changes in the voltage due to the physical properties of the circuit material offers the best environment for this kind of noise. This kind of noise is called multiplicative noise. The model of multiplicative noise can be written as Equation (5):

$$
I(t)=(I-e) S(t)+N(t)
$$

where, $\mathrm{e}=\{0,1\}$ with a probability $\mathrm{p}$ and $\mathrm{I}(\mathrm{t})$ is the resulting data measured at a time $(t), S(t)$ is the original signal measured and $\mathrm{N}(\mathrm{t})$ is the noise introduced by the sampling process, environment and other source of interference. These noises also known as "speckle" noise.

\subsection{D-Discrete Wavelet Transformation}

The Discrete Wavelet Transform (DWT) is a waveform of efficiently limited duration that has no average value zero. Wavelets are compared with sinewave, which are the basis of Fourier analysis. Sinewaves do not have limited duration while wavelets tend to be irregular (Narbada and Bhagwan, 2013). The wavelet decomposition of an image is done as follows: In the first level of decomposition, the image is split into four subbands, namely HH1, HL1, LH1 and LL1, as illustrated in Fig. 2. The HH1 sub-band gives the diagonal details of the image; the HL1 subband gives the horizontal features, while the LH1 represents the vertical structures. The LL1 subband is the low resolution residual consisting of low frequency components. This subband is further split at higher levels of decomposition. It has been shown that the noise standard deviation $\sigma$ can be accurately estimated from the first decomposition level diagonal subband $\mathrm{HH} 1$ by the robust and accurate median estimator (Donoho and Johnstone, 1995b), which was given by Equation (6):

$$
\hat{\sigma}_{\mathrm{w}}=\frac{\operatorname{media}\left(\left|\mathrm{HH}_{1}\right|\right)}{0.6745}
$$

\subsection{Wavelet Based Denoising (WBD)}

The WBD procedure involves three major steps: Forward transformation of the signal to the wavelet domain, wavelet coefficient reduction and transformation of the wavelet coefficients back to the original signal domain. Several fundamental decisions have to be made regarding the selection of the value of the threshold $\lambda$ to distinguish signal and noise, the mother wavelet and the choice of thresholding method, as well as the optimal resolution level or scale for diagnosing. Figure 3 shows the main steps in the denoising algorithm. 


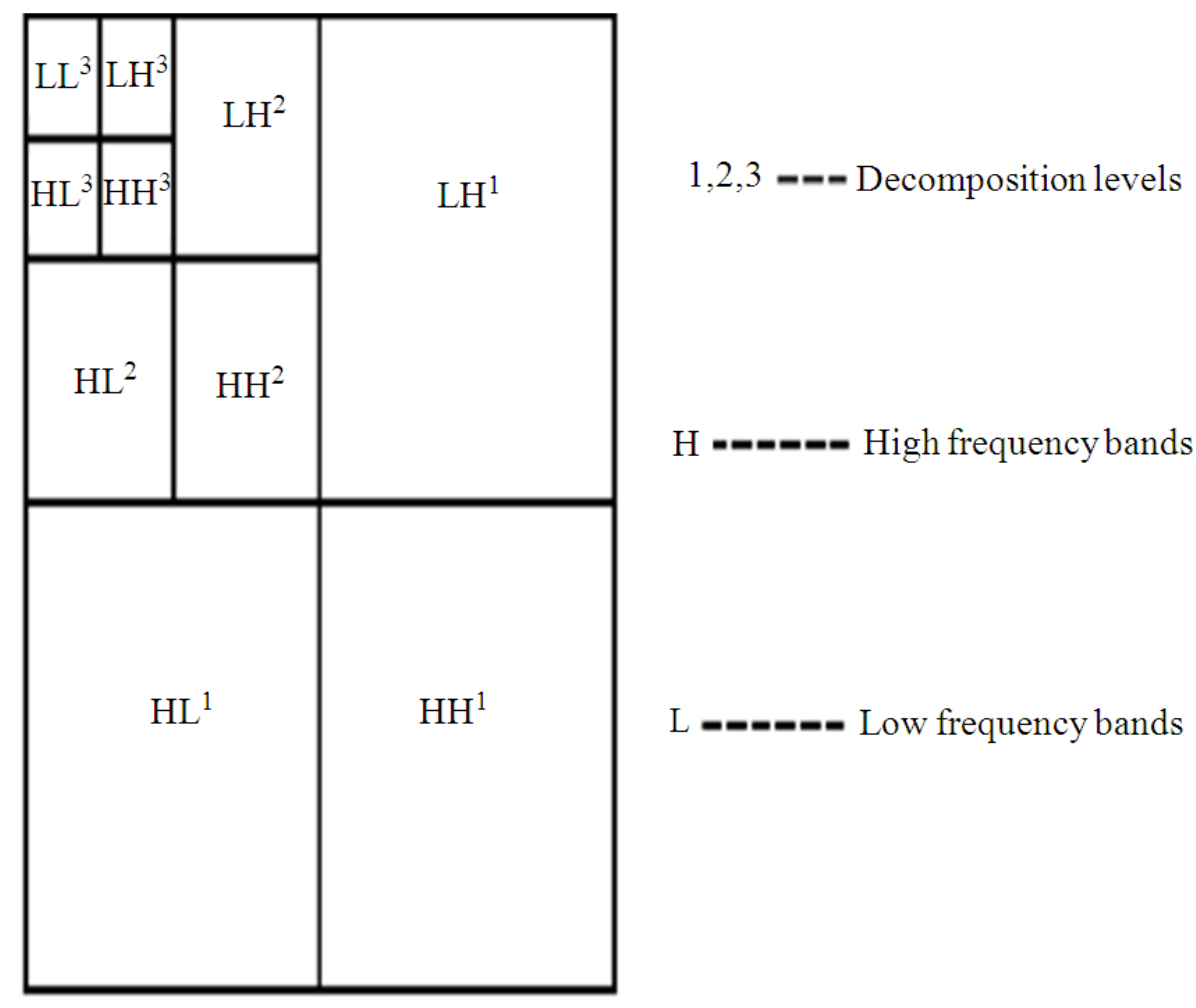

Fig. 2. Subbands of the 2-D orthogonal wavelet transform

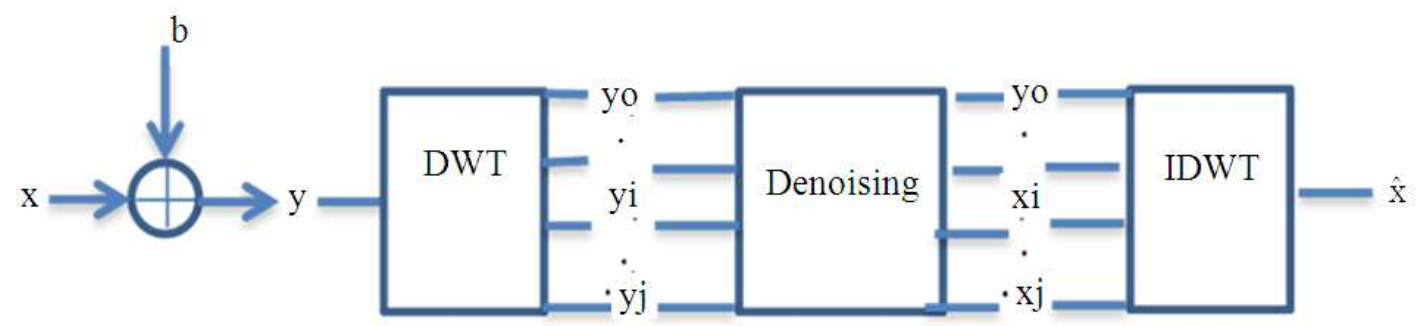

Fig. 3. The main steps of denoising algorithm

\subsection{Hard and Soft Thresholding}

Signal denoising using the DWT consists of the three successive steps namely, signal decomposition, thresholding of the DWT coefficients and signal reconstruction. Firstly, we carry out the wavelet analysis of a noisy signal up to a chosen of decomposition (analysis) level N. Secondly; we perform thresholding of the detail coefficients from the decomposition level 1 to N. Lastly, synthesizing the signal using the altered detail coefficients from level 1 to $\mathrm{N}$ and approximation coefficients of level $\mathrm{N}$
(Misiti et al., 2013). However, it is generally impossible to remove all the noise without corrupting the signal as for thresholding; we can settle either a level-dependent threshold vector of length $\mathrm{N}$ or a global threshold of constant value for all levels according to the method of Donoho and Johnstone (1995b). Thresholding can be either soft or hard. Hard thresholding zeroes out all the signal values smaller than $\lambda$. Soft thresholding does the same thing and apart from that, subtracts $\lambda$ from the values larger than $\lambda$. Soft thresholding causes no discontinuities in the resulting signal. In this study, a new method based on 
semi-soft thresholding is proposed. This method overcomes the drawbacks in both types of thresholding techniques (hard and soft). These drawbacks represented in killing too much coefficients (setting them to zero) in hard thresholding and the over smoothing that affect the reconstructed image in soft thresholding. Figure 4 shows hard and soft thresholding graph that applied on wavelet coefficients.

\subsection{Improving Wavelet Image Denoising via Cycle Spinning}

In spite of the significant developments in WBD, wavelet thresholding methods are not perfect without limitations. Denoising with the traditional wavelet transform (orthogonal, maximally decimated) often show disturbing visual artifacts. In particular, pseudoGibbs artifacts tend to be noticeable in the vicinity of high frequency parts (edges). This is mainly due to the lack of translation invariance of the wavelet basis.

The idea of using "cycle spinning" has been previously proposed for the purpose of minimizing the pseudo-Gibbs disturbing artifacts that are often existing in wavelet-based image reconstruction and denoising (Mohsen, 2004). This can be achieved as follows.

For a range of $\mathrm{K}$ shifts, one shifts of the image with size $\mathrm{M} \times \mathrm{M}$, horizontally or vertically or both, denoises the shifted data using a wavelet thresholding technique of choice and then unshifts the denoised image. Doing

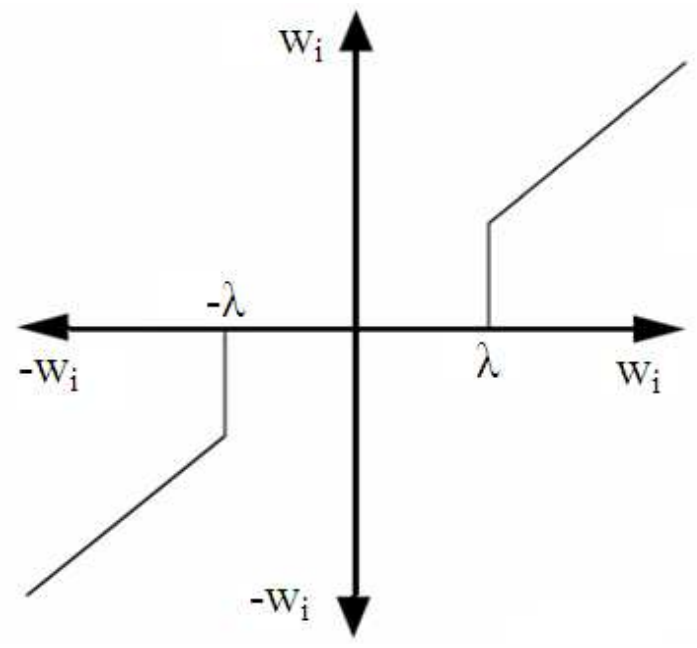

(a) this for each of a range of shifts and averaging the different results so obtained, produces a reconstruction subject to weaker pseudo-Gibbs phenomena than the thresholding-based denoising using the traditional orthogonal wavelet transform.

This is a result of the fact that the discrete wavelet transform is not translation invariant in the case of a periodic signal. In other words, if a periodic signal is shifted, then its wavelet decomposition coefficients are not simply permuted. Mathematical details of this fact have been studied in Mallat (1989).

Since the image is assumed to be periodic with period $\mathrm{M}$, better results can be obtained by using a higher number of shifts $\mathrm{K} \in\{0,1,2, \ldots . \mathrm{M}-1\}$. When $\mathrm{K}$ $=\mathrm{M}-1$, it is said that total-shift cycle spinning is performed. Otherwise only partial-shift cycle spinning is performed. As it will be illustrated, the quality of the denoised signal, as measured by the MSE, PSNR and Quality Indexed Image fidelity measures, improves considerably for the first few values of $\mathrm{K}$. However, for larger values of $\mathrm{K}$, no visible gain is achieved by increasing $\mathrm{K}$ even further. In our experiments $\mathrm{K}$ is chosen to be 8 .

The cycle spinning algorithm may be rather computationally expensive. Indeed, when incorporating this algorithm with $\mathrm{K}$ shifts for any denoising method, the computational complexity is multiplied by $\mathrm{K}$ times.

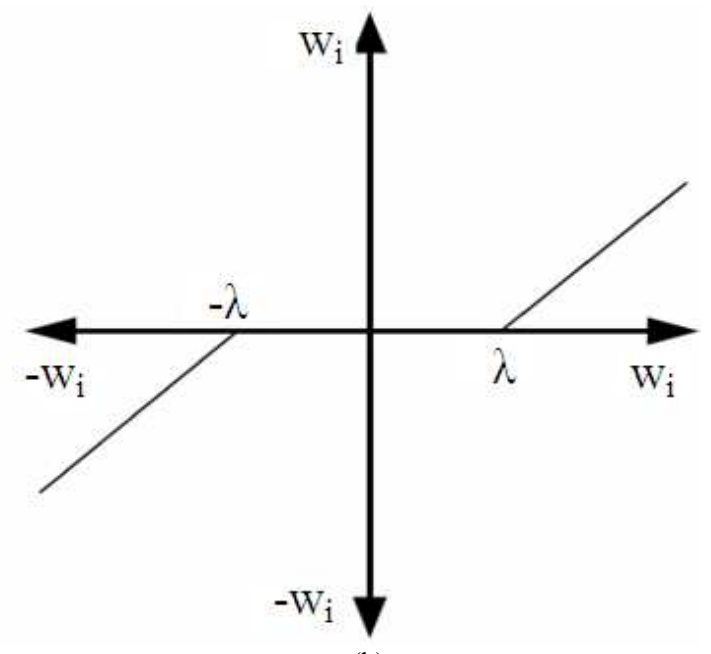

(b)

Fig. 4. (a) Hard thresholding, (b) soft thresholding operators as applied on the wavelet coefficients 


\section{MATERIALS AND METHODS}

A new method that depends on WBD will be presented where wavelet denoising is used with semisoft thresholding and cycle spinning techniques in order to remove the artifacts and noises from the original image.

In this method we derived the thresholding function that depends on the criterion of wavelet coefficient properties and the fact while signal energy becomes more concentrated into few largest coefficients in the transform domain, noise energy concentrated in small coefficients. It helps for the separation of signal from noise. This scheme also yields results that are consistent with the human visual system which is more sensitive to the presence of noise in flat regions than near edges of the image (Mohsen, 2004).

The qualitative measures (i.e., RMSE, PSNR and Quality Index Image) are used in these experiments. This is because; most of the remaining noise is localized in the vicinity of edges and other high-frequency content of the image where the human visual system is less sensitive to noise near edges.

\subsection{Thresholding Function Neighborhood Coefficients}

Using

Wavelet coefficients are correlated in a small neighbourhood. A large wavelet coefficient will probably have large coefficients within its neighbours. Therefore, the proposed thresholding function can be derived from the neighborhood coefficients of the noisy image. Suppose $B_{i, j}$ is the set of wavelet coefficients of the noisy 2D signal. Let Equation (7):

$$
U_{i, j}^{2}=B_{i, j-1}^{2}+B_{i, j}^{2}+B_{i, j+1}^{2}
$$

where, $\mathrm{U}^{2}{ }_{\mathrm{i}, \mathrm{j}}$ is resulted from summation of square of the coefficients where its location in the same row of the coefficient that to be thresholded and $(i, j)$ is representing the location of the coefficient in the contaminated image Equation (8):

$$
\text { If } U_{i, j}^{2} \leq \lambda^{2}
$$

Then we set the wavelet coefficient $B_{i, j}$ to zero. Otherwise, we shrink it according to Equation (9):

$$
B_{i, j}=B_{i, j}^{2}-B_{i, j}^{2} / U_{i, j}^{2}
$$

where, $\lambda=\sqrt{2 \sigma^{2} \log N}, \sigma$ is the noise variance and $N$ is number of pixels in the image (size of the image).

For every (wavelet coefficient) that wanted to be thresholded, a square neighbourhood window $F_{j, k}$ is considered around the coefficient $\mathrm{B}_{\mathrm{i}, \mathrm{j}}$. That means the neighbourhood window size could be represented as $\mathrm{M} \times \mathrm{M}$, where $\mathrm{M}$ is a positive odd number. Figure $\mathbf{5}$ illustrates a $3 \times 3$ neighbourhood window centered at the wavelet coefficient to be thresholded. We should threshold different wavelet coefficient subbands independently because the boundaries of different subbands are not correlated.

Furthermore, when the window size is intermediate, say $3 \times 3$ or $5 \times 5$, the shrinkage to the current wavelet coefficient will take more coefficients in the thresholding function $\mathrm{U}_{\mathrm{i}, \mathrm{j}}^{2}$. Therefore, in this case, more important image features will be kept and more noise will be removed as well. However, if the size of the window around the pixel is too large, a lot of noise will be kept, so an intermediate window size of $3 \times 3$ or $5 \times 5$ is fair to be used. Trade off should be considered when the window size is chosen, because it will affect the quality of the reconstructed image as it was explained early.

\subsection{Wavelet Mother Functions}

Users of the wavelet transform must identify in advance the nature of the type of filter functions that are to be used. These functions will be known as 'mother wavelets functions' and they differ in terms of their smoothing and symmetry properties. The synthetic data were used to assess the effects of the use of a wide range of different mother wavelets (Daubechies, Coiflet and Symmlet). The experience obtained from these experiments allowed the specification of a number of guidelines, which were then used in noise removal (denosing) and compression process in image processing applications. Figure 6 shows the shapes of the mother wavelets investigated in this study.

\subsection{Significance of the Proposed Algorithm}

For speckle noise and Gaussian noise, the image assessment such as PSNR, MSR and Quality indexed image as well as the visual quality give us good results in subjective an objective assessments points of view. In the same regard, the benefits of this research to the community and industry lay in forensic investigation, as well as defense and border security, with the end result of the research being directly applicable in areas of video surveillance and security for analysis and restoration. 


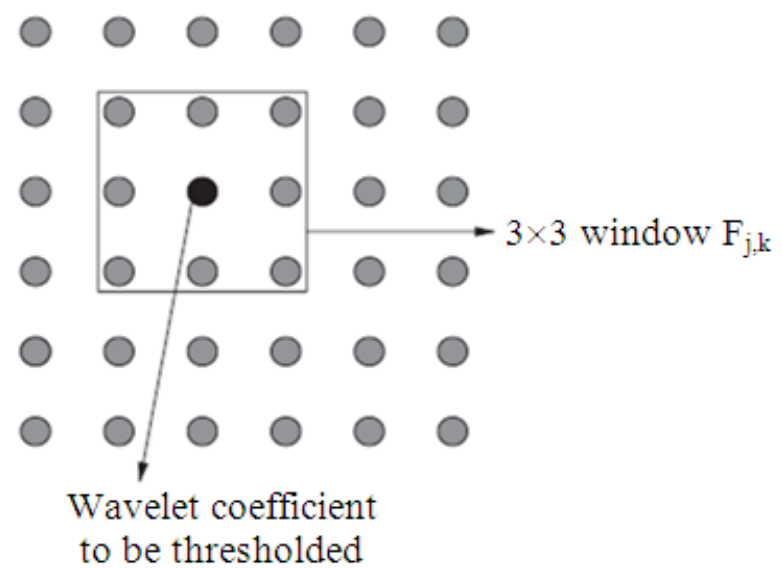

Fig. 5. An illustration of the nieghbourhood window centered at the wavelet coefficient to be thresholded

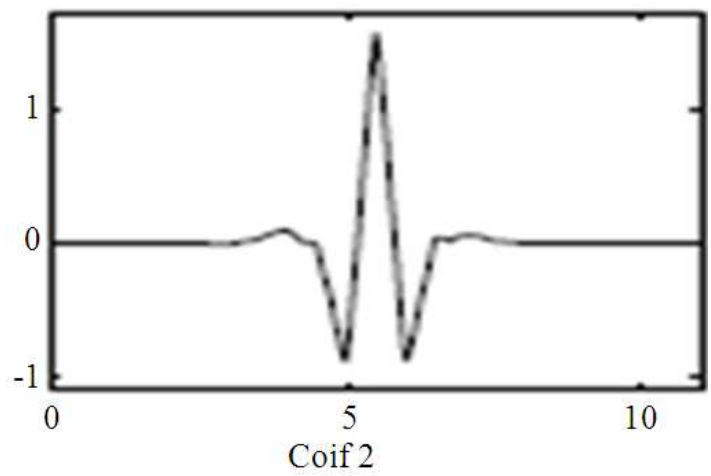

(a)

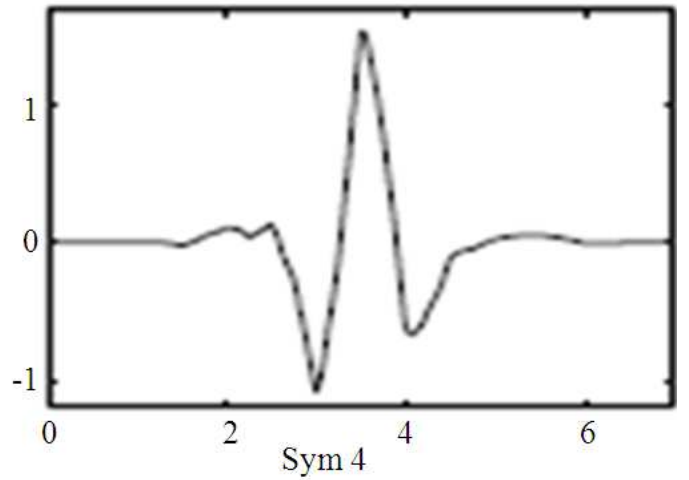

(b)

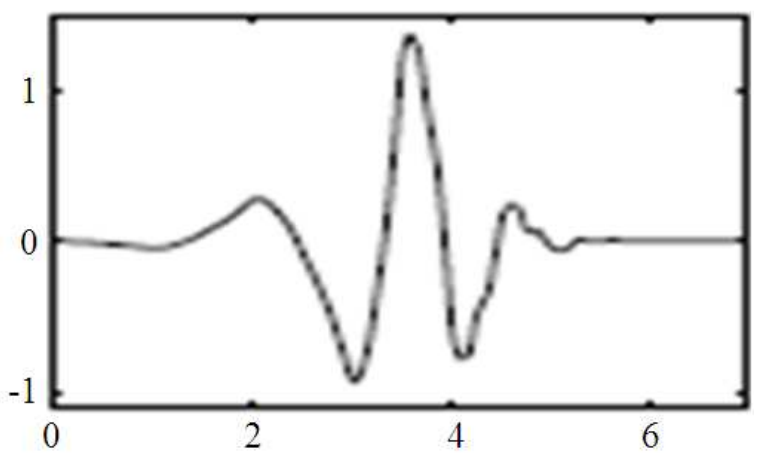

(c)

Fig. 6. The wavelet mother functions that used in this study, (a) Coif2. (b) Sym4. (c) Db4

\section{RESULTS}

In this section, we present numerical experiments to validate the method proposed above. It was applied on a gray scale benchmark image Lena with $512 \times 512$
(Fig. 7). The first experiment was applied on Additive noise (Gaussian), the image has noise variance $\sigma_{\mathrm{n}}=$ 0.05 . For the second experiment, experiment on Spackle noise with noise level $\sigma_{\mathrm{n}}=30$ is conducted. The wavelet transform that we have employed is 
Symmetric supported wavelet with 8 vanishing moments (Sym8) and four scales of orthogonal decomposition. The incorporation of the cycle spinning for the purpose of the denoising based on wavelet transform predictive scheme has resulted in significant improvement of the denoised estimate, as most of the artifacts have been reduced. This has indeed resulted in the best wavelet based denoised estimate.

The performance of noise removing algorithms is measured using quantitative performance measures such as MSE, PSNR and Quality Indexed image as well as in term of visual quality of the images. The mathematical form of the universal quality index $Q$ deals with three main different factors: Loss of correlation, luminance distortion and contrast distortion. $\mathrm{Q}$ is computed for a sliding window of the image size and its highest value is 1 and lowest value is -1 .

The results are given in Table $\mathbf{1}$ and $\mathbf{2}$. It is evident from the tables that our scheme outperforms the standard methods of additive and speckle noise such as VisuShrink, BM3D and the adaptive Median. In other words, our scheme removes noise significantly.

Original image of Lena, Gaussian noisy image and different denoised images with noisy level $\sigma 30$ are shown in Fig. 7. However, Fig. 8 shows the original image, speckle noisy image and different denoised images. The results obtained before and after applying the cycle spinning algorithm are presented.

Figure 7 shows the benchmark image (Lena) with additive noise and the despeckled images. Those filters that mentioned above compared with the proposed method. The best visual results obtained by the proposed method with cycle spinning showed good visual results not only removing speckle but also preserving the details of the image and preserves the edge properties. Flat regions of the image, such as the shoulder, face and background, are relatively smooth most of the noise in these regions have been suppressed in the fractal representation. Also from Fig. 7f, one can see that although the result obtained by wavelet Shrinkage method contains abundant textures, much noise is retained in the image. Figure 7e shows the result obtained by BM3D method. We see that most of the noise have been removed, but many textures are also removed. Figure $7 \mathbf{g}$ and $\mathbf{h}$ show the result by our method with and without cycle spinning respectively, from which we can see that the textures are better preserved while removing the noise, especially in Fig. $\mathbf{7 h}$ the proposed method successfully balances the relationship between noise suppression and texture preserving.

Table 1. Image quality evaluation metrics computed for Lena image with additive noise with $\sigma_{\mathrm{n}}=30$

\begin{tabular}{|c|c|c|c|c|}
\hline \multirow[b]{2}{*}{ Filter method } & \multicolumn{4}{|c|}{ Feature sets } \\
\hline & MSR & PSNR & $\mathrm{Q}$ & Execution time/S \\
\hline Normal shrink & 112.4101 & 28.5241 & 0.3812 & 2.300 \\
\hline BM3D & 84.1201 & 31.2063 & 0.4987 & 1.960 \\
\hline Visu shrink & 91.3778 & 28.1382 & 0.3627 & 3.201 \\
\hline Sure shrink & 87.1179 & 29.6352 & 0.4532 & 43.050 \\
\hline Proposed method & 79.8830 & 31.3654 & 0.5092 & 37.121 \\
\hline Proposed method with CS & 76.4142 & 32.2134 & 0.5218 & 15.400 \\
\hline
\end{tabular}

Table 2. Image quality evaluation metrics computed for Lena image with speckle noise with $\sigma_{\mathrm{n}}=0.05$

\begin{tabular}{|c|c|c|c|c|}
\hline \multirow[b]{2}{*}{ Filter method } & \multicolumn{4}{|c|}{ Feature sets } \\
\hline & MSR & PSNR & Q & Execution time/S \\
\hline Lee filter & 165.6337 & 25.9393 & 0.4474 & 24.741319 \\
\hline Median & 263.3980 & 23.9247 & 0.4534 & 0.780064 \\
\hline Weiner & 148.6431 & 26.4094 & 0.4357 & 0.732820 \\
\hline Lsmv filter & 112.2887 & 27.0114 & 0.4645 & 3.100400 \\
\hline Proposed method & 97.8831 & 28.2237 & 0.4850 & 15.112100 \\
\hline Proposed method With CS & 88.1524 & 28.6785 & 0.4962 & 11.019210 \\
\hline
\end{tabular}




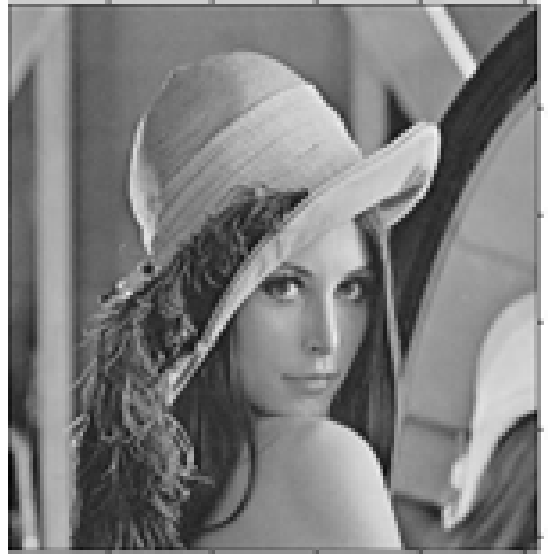

(a)

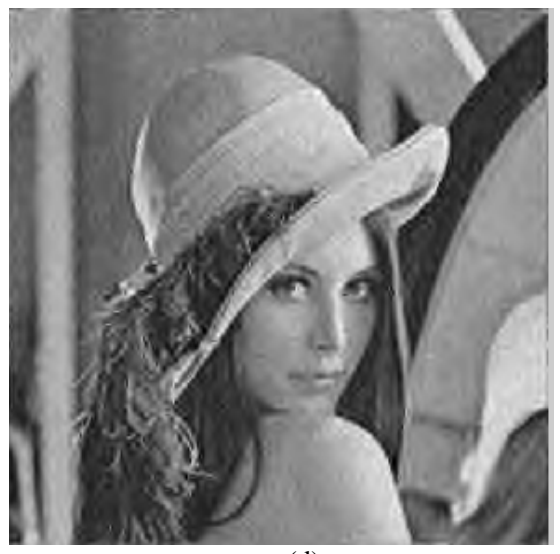

(d)

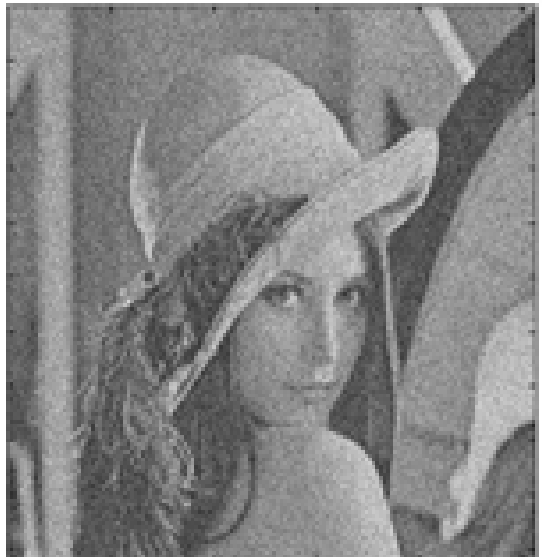

(b)

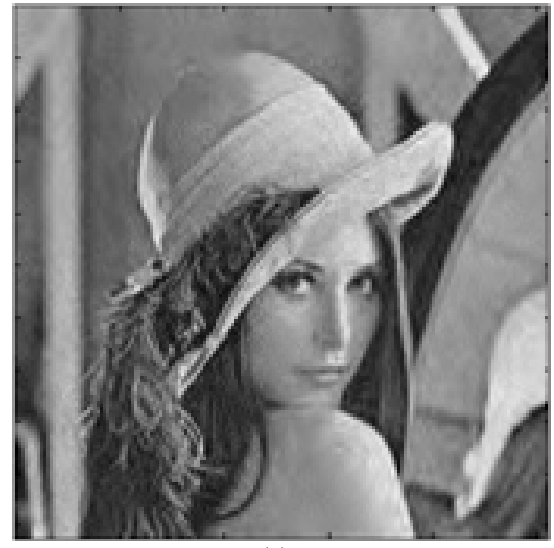

(e)

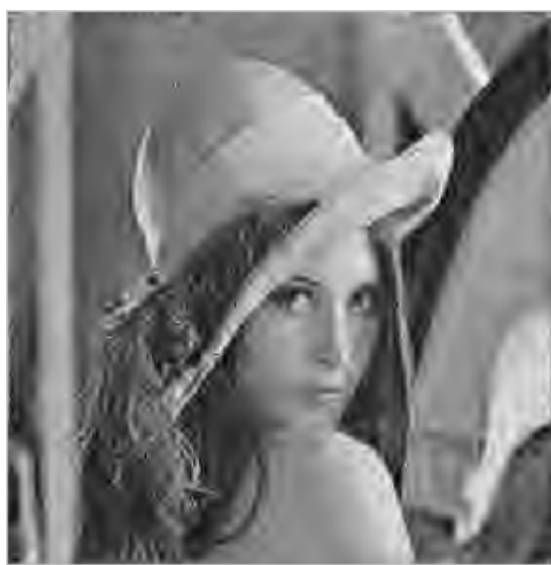

(c)

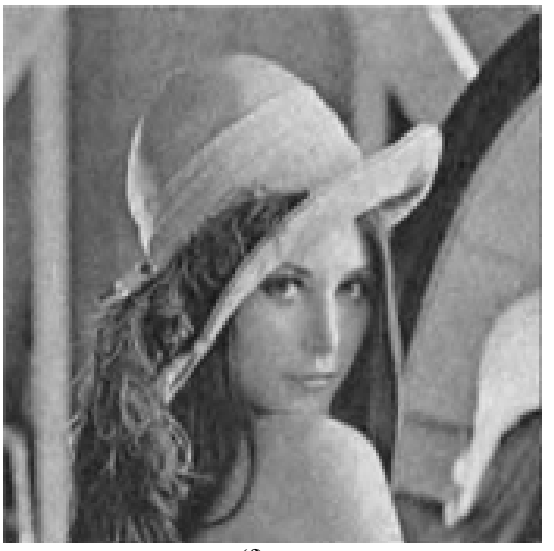

(f)

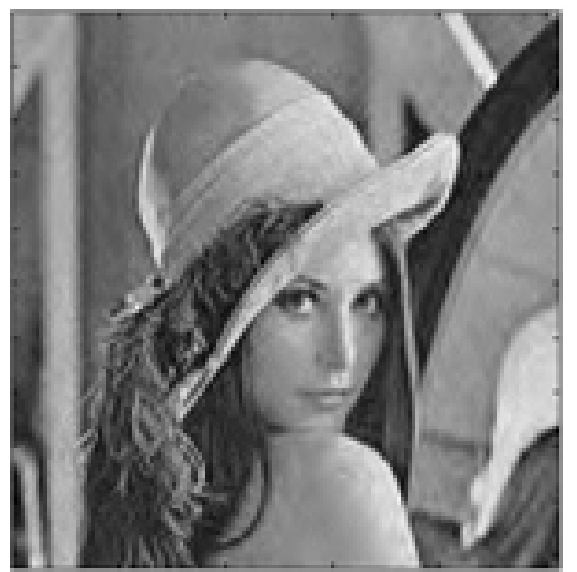

(g)

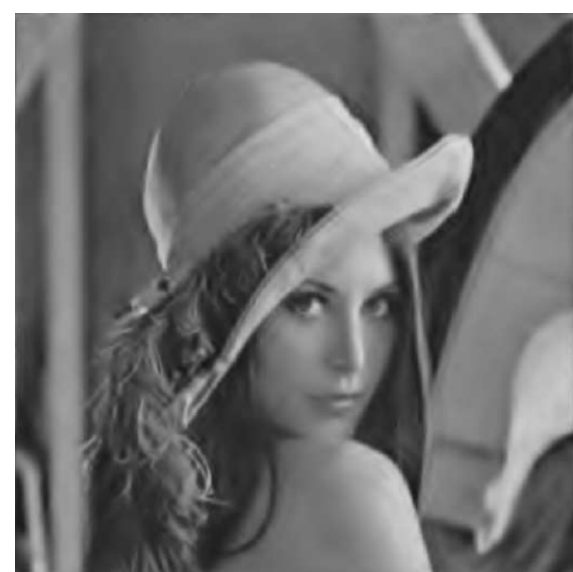

(h)

Fig. 7. Results of various filters on an additive noise with $\sigma n=30$, (a) Original image (Lena image), (b) Noisy image (c) Visushrink filter, (d) Sureshrink filtering, (e) BM3D filter, (f) Normal shrink filter, (g) Proposed method, (h) Proposed method using SC with $\mathrm{K}=8$ shifts 


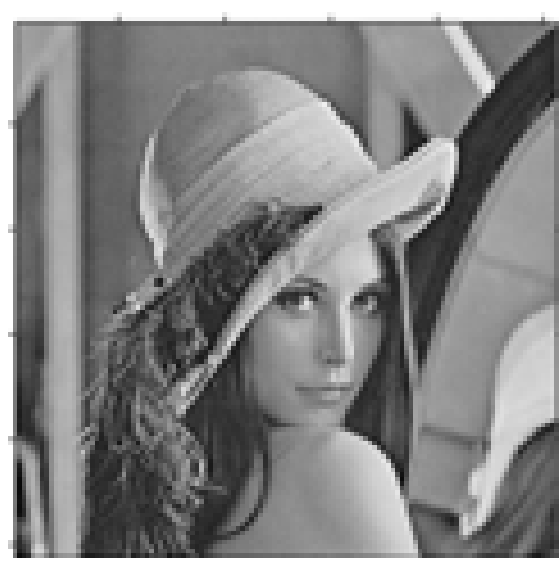

(a)

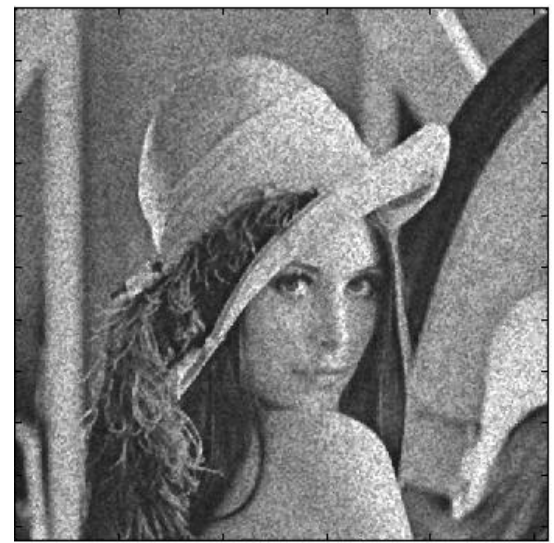

(d)

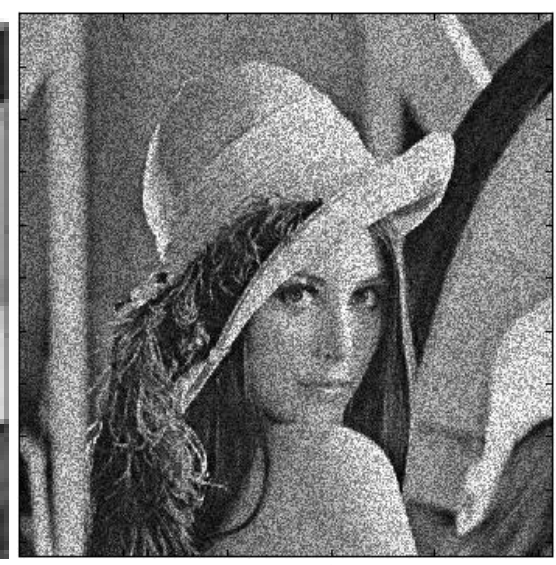

(b)

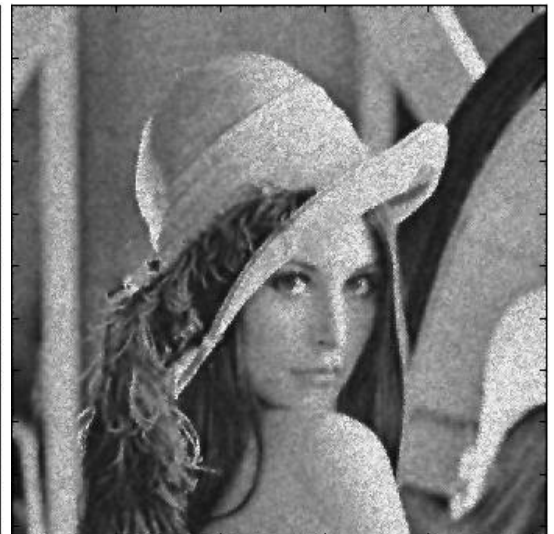

(e)

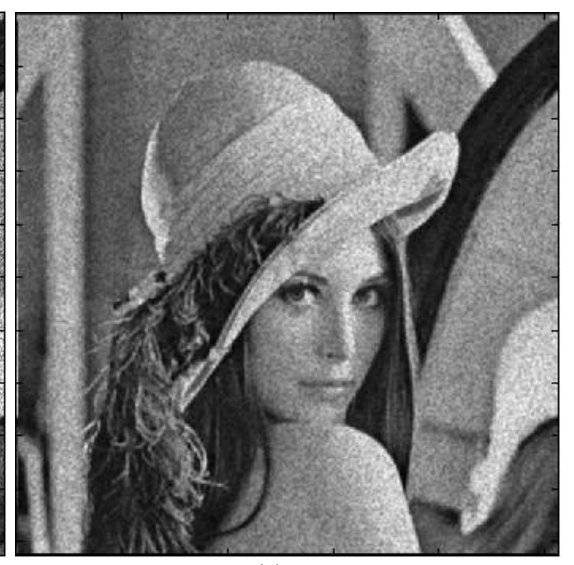

(c)

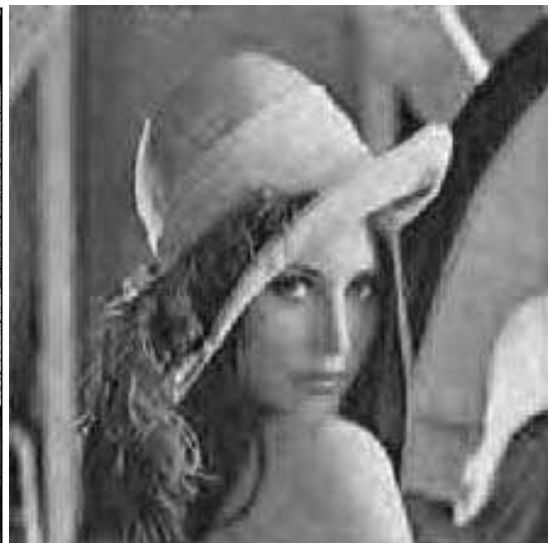

(f)

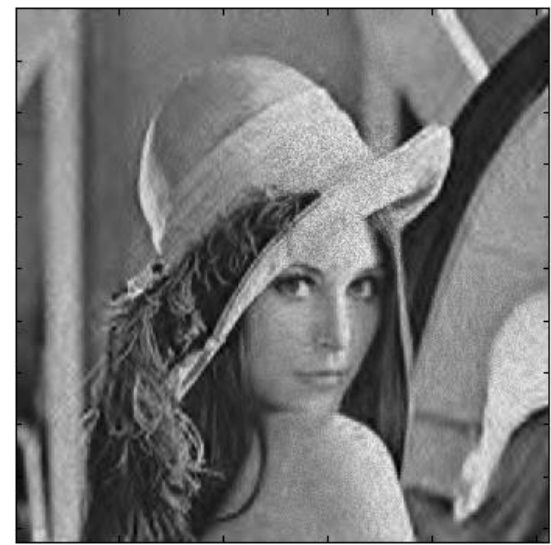

(g)

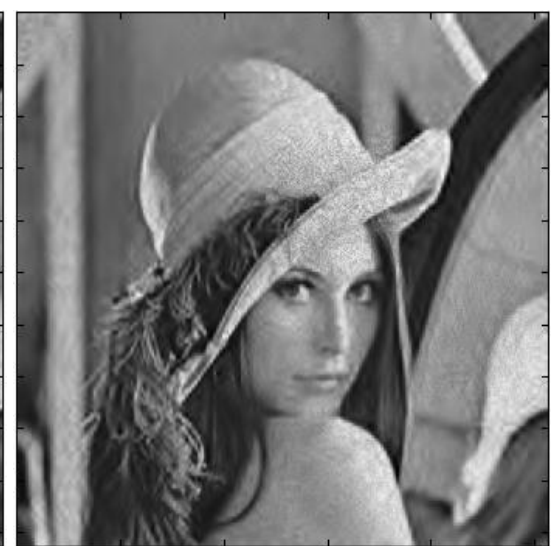

(h)

Fig. 8. Results of various filters on a multiplicative noise with $\sigma n=0.05$, (a) Original image (Lena image), (b) Noisy image, (c) Lee filter, (d) Median filter, (e) Wiener filter,(f) Lsmv filter, (g) Proposed method, (h) Proposed method using SC with K = 8 shifts

Benchmark image (Lena) with multiplicative (speckle) noise and the despeckled images are shown in
Fig. 8. The best visual results obtained by the proposed method with cycle spinning and 8 shifts showed good 
visual results by not only removing speckle but also preserving the details of the image especially in the edges where the high frequency appears. Also from the Fig. 8e, it is clear to see that although the result obtained by wavelet shrinkage method contains too much textures and image details, highly noise is retained in the image.

The result obtained by Lsmv filter is shown in Fig. 8f. We see that most of the noise have been removed, but many textures are removed as well. Figure $8 g$ and $h$ show the result by our method with and without cycle spinning respectively. It is evident to notice that the textures are better preserved while removing the noise, especially in (h) where we use the proposed method with cycle spinning $(\mathrm{K}=8)$. The proposed method successfully balances the relationship between noise suppression and texture preserving.

\section{DISCUSSION}

Table 1 shows the image quality evaluation metrics, Lena image between the original and the despeckled image, respectively. Performance of all additive noise algorithms is tested with the bench mark image. The number of shifts $\mathrm{K}$ that should be taken should be less than the size of the image $\mathrm{N}$ i.e., $(\mathrm{K} \leq \mathrm{N})$. Best values were obtained for the proposed method with cycle spinning with shift $\mathrm{K}=8$, it gives lower MSE and higher SNR and PSNR and best values for the universal Quality index (Q), Filters Normal shrink, Visushrink, Sure shrink, showed poorer visual results and a blurring effect. But the filters of the proposed algorithm and BM3D show better visual results compared to these filters. In addition, the execution time plays a major role especially when programming time and running time are taken into consideration. In our experiments it is clear to notice that BM3D has the shortest execution time compared with the rest of other filters, while Sureshrink has the longest one. Our method has an average time lies in the range of 15-37 sec. It has been found that the proposed method with CS is better than all other algorithms in the quantitative terms as well as the visual quality of the image.

Table 2 shows the image quality evaluation metrics, Lena image between the original and the despeckled image, respectively. Performance of all speckle noise algorithms was tested with the bench mark image. Best values were obtained for the proposed method with cycle spinning with shift $\mathrm{K}=8$, it gives lower MSE, higher PSNR. Best values for the universal Quality index (Q), Filters Lee, Lsmv while Median showed poorer visual results and a blurring effect. But the filters of the proposed algorithm and Weiner show better visual results compared to these filters. Moreover, in terms of execution time; it is clear to notice that Weiner filter has the shortest time compared with the rest of other filters where it is considered as the sharpest and standard filter in multiplicative noise. On the other hand, Lee filter is the worst filter in the execution time because of the construction of its functions. It has been found that the proposed method with CS is better than all other filters in quantitative terms as well as visual quality of the image.

\section{CONCLUSION}

In this study, we proposed a speckle and additive Gaussian noise reduction method by combining a wavelet-based denoising shrinkage filter and cycle spinning with $\mathrm{K}$ shifts. The proposed hybrid method takes full advantage of sparsity and orthogonality in wavelet with the cycle spinning technique to denoise the low frequency subband without losing textures and uses the wavelet shrinkage method based on local variance information to extract textures from noise in the high frequency subbands. The image denoising algorithm uses semi-soft thresholding to provide smoothness and better edge preservation at the same time. Not only removing speckle and additive noise, but also preserving the details of the image and is better than all other algorithms in quantitative terms as well as visual quality of the image.

Two experiments have been conducted on the bench mark image (Lena), the first experiment is conducted on additive white Gaussian noise to compare our method with many other well-known techniques such as the VisuShrink, Normal shrink, BM3D and Sureshrink. The results show that our method outperformed the traditional methods and removes noise significantly most of the time. In the second experiment, it was conducted on multiplicative noise (Speckle), our method compared with Lee filter, Median filter, Wiener and Lsmv filter. The results show that the proposed method with cycle spinning with $K=8$ shifts outperformed the method as it was mentioned earlier and gave better preservation where most of details in the denoised image are kept clear. On the other hand, the most important parameter in our experiments that computed is the execution time. It shows that the standard methods like Wiener, Median and BM3D filters give the shortest time in this experiment. The use of cycle spinning causes for the slowing of the running time compared with the standard filters. In the future work, we will work on the enhancement of the running time in order to reach to the 
same level of the quality image that is got from this study. Our experiments are implemented on a PC (CPU: Intel core i7 $9503.07 \mathrm{GHz}, 3 \mathrm{~GB}$ Ram).

\section{REFERENCES}

Aujol, F. and A. Chambolle, 2005. Dual norms and image decomposition models. Int. J. Comput. Vis., 63: 85-104. DOI: 10.1007/s11263-005-4948-3

Balaiah, P. and Ilavennila, 2012. Comparative evaluation of adaptive filter and neuro-fuzzyfilter in artifacts removal from electroencephalogram signal. Am. J. Applied Sci., 9: 1583-1593. DOI: 10.3844/ajassp.2012.1583.1593

Banazier, A. and K. Yasser, 2011. Speckle noise reduction method combining total variation and wavelet shrinkage for clinical ultrasound imaging. Proceedings of the 1st Middle East Conference on Biomedical Engineering, Feb. 21-24, IEEE Xplore Press, Sharjah, pp: 80-83. DOI: 10.1109/MECBME.2011.5752070

Chambolle, A., 2004. An algorithm for total variation minimization and applications. J. Math. Imag. Vis., 20: 89-97. DOI: 10.1023/B:JMIV.0000011325.36760.1e

Chambolle, A., R. Devore and N. Lee, 1998. Nonlinear wavelet image processing variational problems, compression and noise removal through wavelet shrinkage. IEEE Trans. Image Process., 7: 319-335. DOI: $10.1109 / 83.661182$

Chang, S., B. Yu and M. Vetterli, 2000. Adaptive wavelet thresholding for image denoising and compression. IEEE Trans. Image Process., 9: 15321546. DOI: $10.1109 / 83.862633$

Daubechies, I., 1990. The wavelet transform timefrequency localization and signal analysis. IEEE Trans. Inform. Theory, 36: 961-1005. DOI: 10.1109/18.57199

Donoho, D.L. and I.M. Johnstone, 1994. Ideal spatial adaptation by wavelet shrinkage. Biomerika J., 81: 425-455. DOI: 10.1093/biomet/81.3.425

Donoho, D.L. and I.M. Johnstone, 1995a. Adapting to unknown smoothness via wavelet shrinkage. J Am. Stat. Assoc., 90: 1200-1224.

Donoho, D.L. and I.M. Johnstone, 1995b. Denoising by soft thresholding. IEEE Trans. Inform. Theory, 41: 613-627. DOI: 10.1109/18.382009

Durand, S. and J. Froment, 2003. Reconstruction of wavelet coefficients using total variation minimization. SIAM J. Scientific Comput., 24: 1754-1767.
Gilboa, G., N. Sochen and Y.Y. Zeevi, 2006. Texture preserving variational denoising using an adaptive fidelity term. IEEE Trans. Image Process., 15: 22812289. DOI: 10.1109/TIP.2006.875247

Hsung, T., D.B.K. Lun and K.C. Ho, 2005. Optimizing the multiwavelet shrinkage denoising. IEEE Trans. Signal Process., 53: 240-250. DOI: 10.1109/TSP.2004.838927

Khmag, A., A.R. Ramli, S.J. Hashim and S.A.R. AlHaddad, 2013. Review of image denoising algorithms based on the wavelet transformation. Int. J. Adv. Trends Comput. Sci. Eng., 2: 01-08.

Ladan, E., H.Z.M. Shafri, B.M. Shattri and A. Ravshan, 2013. A review of applying second-generation wavelets for noise removal from remote sensing data. Environ. Earth Sci., 70: 2679-2690. DOI: 10.1007/s12665-013-2325-z

Luisier, F., T. Blu and M. Unser, 2007. A new SURE approach to image denoising: Interscale orthonormal wavelet thresholding. IEEE Trans. Image Process., 16: 593-606. DOI: 10.1109/TIP.2007.891064

Mallat, S.G., 1989. A theory for multiresolution signal decomposition: The wavelet representation. IEEE Trans. PAMI, 11: 674-693. DOI: $10.1109 / 34.192463$

Meyer, Y., 2002. Oscillating Patterns in Image Processing and Nonlinear Evolution Equations. 1st Edn., American Mathematical Society Press, Providence, ISBN-10: 0821829203, pp: 122.

Misiti, M., Y. Misiti, G. Oppenheim and J.M. Poggi, 2013. Wavelet toolbox. MathWorks, Inc.

Mohsen, G., 2004. Adaptive fractal and wavelet image denoising. University of Waterloo Library.

Narbada, S. and S. Bhagwan, 2013. Image denoising using biorthonormal wavelet transforms using Stein unbiased risk estimator. Int. J. Emerg. Trends Electron. Comput. Sci., 2: 6-10.

Osher, S., A. Sole and L. Vese, 2003. Image decomposition and restoration using total variation minimization and the $\mathrm{H}-1$ norm. J. Multiscale Model. Simulat., 1: 349-370. DOI: 10.1109/ICIP.2003.1247055

Portilla, J., V. Strela, M.J. Wainwright and E.P. Simoncelli, 2003. Imagedenoising using scale mixtures of Gaussians in the wavelet domain. IEEE Trans. Image Process., 12: 1338-1351. DOI: 10.1109/TIP.2003.818640 
Qing-Wu, L., N. Xue and D. Shi, 2007. Image denoising method using multiple wavelet bases and fusion technology. Opto-Electron. Eng., 34: 103-107.

Rudin, L., S. Osher and E. Fatemi, 1992. Nonlinear total variation based noise removal algorithms. Phys. J., 60: 259-268. DOI: 10.1016/01672789(92)90242-F

Sankar, A.B., D. Kumar and K. Seethalakshmi, 2012. A new self-adaptive neuro fuzzy inference system for the removal of non-linear artifacts from the respiratory signal. J. Comput. Sci., 8: 621-631. DOI: 10.3844/jcssp.2012.621.631
Shamik, T., K. Ajay and V.P. Shukla, 2011 Statistical moments based noise classification using feed forward back propagation neural network. Int. J. Comput. Applic., 18: 36-40. DOI: 10.5120/2254-2886

Suganthy, M. and P. Ramamoorthy, 2012. Principal component analysis based feature extraction, morphological edge detection and localization for fast iris recognition. J. Comput. Sci., 8: 1428-1433. DOI: $10.3844 /$ jcssp.2012.1428.1433

Yasmin, M., M. Sharif, S. Masood, M. Raza and S. Mohsin, 2012. Brain image enhancement-a survey. World Applied Sci. J., 17: 1192-1204. 